\title{
Etude comparée des potentialités hivernales d'assimilation carbonée de trois conifères de la zone tempérée (Pseudotsuga menziesii Mirb., Abies alba Mill. et Picea excelsa Link.)
}

\author{
J.M. GUEHL \\ avec la collaboration technique de B. CLERC et J.M. DESJEUNES \\ I.N.R.A., Station de Sylviculture et de Prodaction \\ Centre de Recherches forestières de Nancy, Champenoux, F 54280 Seichamps
}

\section{Résumé}

L'évolution au cours de l'hiver de lactivité photosynthétique de rameaux de douglas, sapin pectiné et d'épicéa provenant de plantations est étudiée.

La relation entre la photosynthèse nette et la température actuelle est similaire pour les 3 cspèces avec un large domaine optimal de 5 à $25^{\circ} \mathrm{C}$. Les points inféricurs de compensation thermique de la photosynthèse sont de $-3^{\circ} \mathrm{C}$ pour le douglas et $-5^{\prime \prime} \mathrm{C}$ pour les autres espèces.

Pour les 3 espèces, on note un scuil thermique de dépression rémanente de la photosynthèse voisin de $-1,-2{ }^{\circ} \mathrm{C}$. Les variations au cours de l'hiver de la capacité photosynthétique sont entièrcment expliquées par celles des résistances internes à la diffusion du $\mathrm{CO}_{2}$.

Le douglas et le sapin pectiné, espèces originaires de zones à hivers relativement doux et dont la résistance au gel est limitée, présentent une photosynthèse hivernale uniquement affectée, et de façon parfaitement réversible, par les variations accidentelles liées aux stress thermiques.

La provenance d'ćpicéa étudiée, d'origine continentale et probablement extrêmement résistante au froid, révèle un modèle d'évolution dans lequel une tendance saisonnière de dépression hivernale de la photosynthèse sc superpose aux variations accidentelles.

Une discussion est engagée tendant à montrer que chacun de ces deux types de comportements pourrait être généralisé en fonction du type de résistance au gel.

\section{Introduction}

Les espèces de conifères originaires des franges côtières océaniques de la zone tempérée sont capables de photosynthétiser de façon importante en hiver dans des conditions climatiques ne s'éloignant pas trop de celles de leur aire d'origine (FRY \& Phillips, 1977, Gulhi, 1982). 
Dans ces mêmes conditions climatiques favorables, la plus grande résistance au froid des espèces originaires de zones climatiques plus continentales ou montagnardes (LARCHER, 1981; SAKAI, 1983), où la photosynthèse hivernale est quasi-inexistante (Zelawski \& Goral, 1966 ; Tranouillini, 1979), s'accompagne-t-elle de potentialités d'assimilation carbonée hivernale plus grandes? ou au contraire ces potentialités sont-elles plus faibles en raison de l'existence d'un rythme endogène saisonnier d'activité fixé génétiquement (LARCHer, 1981; SCHWARz, 1970, 1971)?

Afin d'apporter des éléments de réponse à cette question nous avons entrepris l'étude comparative des échanges gazeux hivernaux de $\mathrm{CO}$., de 3 espèces de conifères : le douglas vert (Pseudotsuga menziesii Mirb.), espèce originaire de la frange côtière pacifique de l'Amérique du Nord; le sapin pectiné (Abies alba Mill.), espèce à tendance continentale et montagnarde, mais dont la provenance étudiée ici (Normandie, Ouest de la France) possède des traits océaniques certains; l'épicéa (Piceat excelsa Link.), espèce continentale et montagnarde s'étendant jusque dans les domaines subarctique et subalpin de l'Hémisphère Nord et caractérisée par une très grande résistance au gel hivcrnal (SAKAI, 1983).

\section{Matériel et méthodes}

Les échanges gazeux de $\mathrm{CO}_{2}$ et $\mathrm{H}_{2} \mathrm{O}$ ont été mesurés sur des rameaux coupés prélevés dans des plantations situées à proximité du Centre National de Recherches forestières de Nancy dans le Nord-Est de la France (altitude $250 \mathrm{~m}$ ). Les espèces étudiées étaient :

- le douglas, provenance Seaquest, état du Washington aux U.S.A., lat. $48^{\prime \prime} 25^{\prime} \mathrm{N}$, long. $121^{\prime \prime} 35^{\prime} \mathrm{W}$, alt. $100 \mathrm{~m}$, âge de la plantation 23 ans ;

- le sapin pectiné, provenance Ecouves, lat. $48^{\prime \prime} 5^{\prime} \mathrm{N}$, long. $0^{\prime \prime}$, alt. $400 \mathrm{~m}$, âge de la plantation 15 ans;

- l'épicéa commun, provenance Istebna en Pologne, alt. 500 à $700 \mathrm{~m}$, âge de la plantation 12 ans.

Pour chacune des journées de mesure (périodicité généralement hebdomadaire) de l'hiver 1982-1983, trois rameaux de l'année de chaque espèce étaient prélevés entre 1 et $2 \mathrm{~m}$ de hauteur sur la bordure extérieure des peuplements. L'extrémité coupée était mise sous eau immédiatement après section et les rameaux étaient transportés en laboratoire où l'on a mesuré leur intensité de photosynthèse nette $\left(\mathrm{F}_{\mathrm{N}}\right.$, $\mathrm{mg} \mathrm{CO} . \mathrm{dm}^{-2} \cdot \mathrm{h}^{-1}$ ) et de transpiration ( $\mathrm{TR}, \mathrm{g} \mathrm{H}_{2} \mathrm{O} \cdot \mathrm{dm}^{-2} \cdot \mathrm{h}^{-1}$ ) à l'aide d'un système de mesure de type Sirigor-Siemens décrit par Ducrey (1981 a). Sauf spécifications contraires, les résultats dont il sera rendu compte concernent des mesures faites dans les conditions standardisées suivantes : concentration en $\mathrm{CO}_{2}$ de l'air égale à $300 \mathrm{vpm}$, température de l'air 20 "C, 80 p. 100 d'humidité relative, éclairement incident foumi par une lampe à vapeur de sodium haute pression SONT correspondant à une densité de flux de photons de $460, \mathrm{E} . \mathrm{m}^{-2^{2}} \cdot \mathrm{s}^{-1}$. Nous avons pu établir que ce niveau d'éclairement est saturant ou quasi-saturant pour la photosynthèse des espèces étudiées ici, du moins en hiver. Divers essais expérimentaux menés dans notre laboratoire tendent à montrer que la méthode des rameaux coupés donne acccès, dans les conditions d'humidité atmosphérique de l'expérimentation, à un potentiel de photosynthèse caracté- 
risant l'optimisation des facteurs hydriques ou moment de la mesure. Cette méthode permet en outre l'étude en laboratoire d'un matériel végétal ayant une histoire climatique naturelle.

La résistance stomatique $\left(\mathrm{r}_{*}, \mathrm{~s}, \mathrm{~cm}^{-1}\right)$ à la diffusion $\mathrm{du} \mathrm{CO}$, a été déterminée à partir des mesures de transpiration comme suit :

$$
r_{s}=\left|\frac{C^{\prime} f-C^{\prime} a}{T R} .36-r_{i 1}\right| \cdot u
$$

où $\mathrm{C}^{\prime} \mathrm{f}\left(\mathrm{g} \cdot \mathrm{m}^{-3}\right)$ est la concentration saturante en vapeur d'eau à la température de feuille supposée être égale à la température de l'air (rayonnement à faible proportion d'infra-rouge, ventilation interne de l'enceinte de $1 \mathrm{~m} . \mathrm{s}^{-1}$ ):

C'a est la concentration en vapeur d'eau de l'air dans l'enceinte d'assimilation ; TR est le taux de transpiration ( $\mathrm{g} \mathrm{H}_{2} \mathrm{O} . \mathrm{dm} \cdots \mathrm{h}^{-1}$ );

$r_{i}$ est la résistance de la couche limite supposée être ćgale à $0,3 \mathrm{~s} . \mathrm{cm}^{-1}$ pour les conifères étudiés (LANDSBERg \& LUDLOW, 1970);

a est le rapport du coefficient de diffusion de la vapeur d'eau à celui du CO. $(\alpha=1,57)$.

La résistance interne à la diffusion du $C O_{2}\left(\mathrm{r}_{\mathrm{i}}, \mathrm{s}, \mathrm{cm}^{-1}\right)$, somme de la résistance au transfert du $\mathrm{CO}_{2}$ des espaces intercellulaires aux sites de carboxylation dans les chloroplastes et de la résistance biochimique à la carboxylation a été calculée d'après la formule suivante dans l'hypothèse, dont on trouvera une justification au $\$ 3.2 .3$, d'une limitation de la photosynthèse par les processus de diffusion du CO.. (Chartier \& BETHENOD, 1977) :

$$
r_{i}=\frac{\mathrm{Ca}-\mathrm{I}^{\prime}}{\mathrm{F}_{\mathrm{x}}} \cdot 0,72-\left(\mathrm{r}_{\mathrm{s}}+\mathrm{r}_{\mathrm{a}}\right)
$$

où Ca est la teneur en $\mathrm{CO}_{2}$ de l'air dans l'enceinte d'assimilation (300 vpm);

$\mathrm{I}$ 'est la valeur du point de compensation de la photosynthèse pour $\mathrm{lc} \mathrm{CO}_{2}$ (vpm) ;

$\mathrm{F}_{X}$ est la densité du flux de photosynthèse nette $\left(\mathrm{mg} \mathrm{CO} . \mathrm{dm} \div . \mathrm{h}^{-1}\right)$ dans laquelle est également intégrée la respiration mitochondriale des parties non vertes des rameaux qui reste en hiver et en dehors de la période de débourrement, faible par rapport à lá photosynthèse en lumière saturante.

Pour l'ensemble des déterminations de $r_{i}$ on a adopté la valeur de $\Gamma^{\prime}=40 \mathrm{vpm}$ trouvée à $20^{\circ} \mathrm{C}$ par NeILson et al. (1972) pour l'épicéa de Sitka. L'hypothèse de constance de $\Gamma$ au cours de l'hiver est un passage obligé pour la détermination de $r_{i}$ en l'absence de connaissances plus précises. Notons toutefois qu'un calcul d'erreurs simple montre que des fluctuations de $30 \mathrm{vpm}$ de part et d'autre de la valeur $I^{\prime}=40 \mathrm{vpm}$ n'entraineraient que des variations relatives de 15 p. 100 autour des estimations de $r_{i}$. On vérifiera dans la suite que les résultats concernant l'importance relative de $r_{s}$ et $r_{i}$ ne se ressentiront pas de telles variations. 


\section{Résultats}

\subsection{Relations entre la photosynthèse nette $\left(\mathrm{F}_{X}\right)$ et la température $(\theta)$}

On considérera dans une premier temps uniquement les courbes de la figure 1 établics en hiver (Mars 1983) qui ne montrent pas, au-dessus de 0 " $\mathrm{C}$, de différences significatives entre espèces. L'optimum thermique est atteint à $20^{\circ} \mathrm{C}$ pour le sapin et le douglas et semble plus proche de 15 "C pour l'épicéa, mais la caractéristique principale des courbes est constituée par la très faible sensibilité à la température dans un domaine très large $\left(5-25^{\circ} \mathrm{C}\right)$.

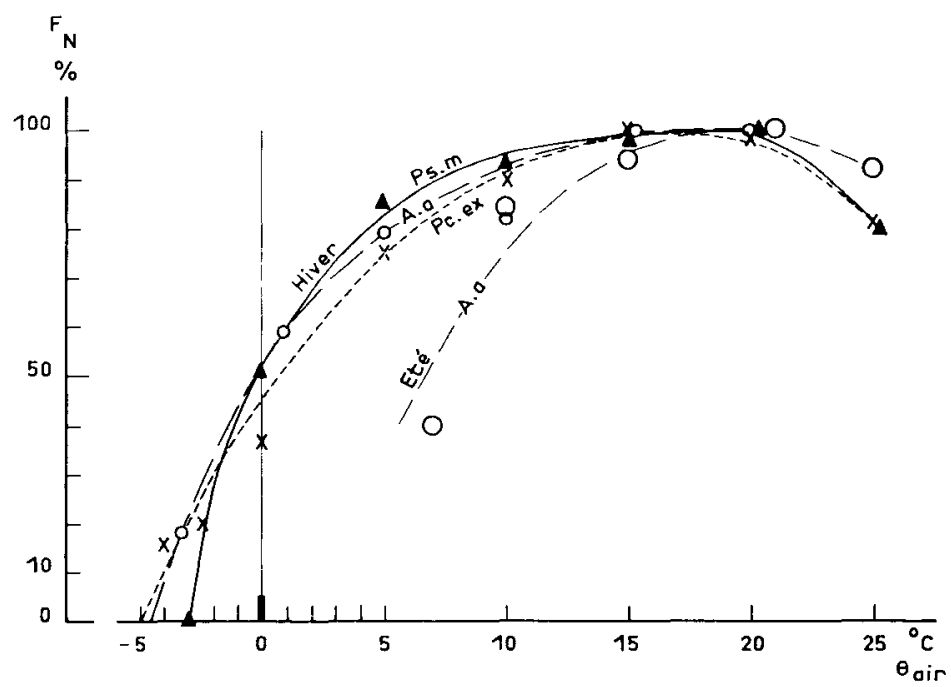

FIG. I

Relations entre la photosynthèse nette et la température de l'air pour le douglas (Ps.m), le sapin (A.a.) et l'épicéa (Pc.ex) en hiver;

ane courbe concerne la photosynthèse du sapin en été.

Relationship between net photosynthesis $\left(F_{x}\right)$ and air temperature $(\theta)$ for douglas-fir (Ps.m), silver-fir (A.a.) and Norway spruce (Pc.e. $)$ in winter and in summer (only for silver-fir).

A 0 "C l'intensité photosynthétique est encore voisine de 50 p. 100 du maximum pout les 3 espèces. Le point de compensation thermique inférieur de la photosynthèse nette, égal à -3 "C pour le douglas, serait d'après l'extrapolation des courbes voisin de $-5^{\circ} \mathrm{C}$ pour le sapin et l'épicéa.

La courbe établie en été (septembre 1983) pour le sapin est très nettement différente de celle obtenue en hiver. $F_{X}$ est affecté très fortement par les basses températures inférieures à 10 " $\mathrm{C}$ et la chute de $\mathrm{F}_{X}$ semble moins accentuée vers les 
forles températures $\left(20-25^{\circ} \mathrm{C}\right)$, sous réserve toutefois d'une diminution hivernale pour le sapin dans ce domaine thermique analogue à celle notée pour les deux autres espèces (pas de mesure à $25^{\circ} \mathrm{C}$ pour le sapin en hiver).

\subsection{Variations de la capacité photosynthétique au cours de l'hiver}

On désigne par capacité photosynthétique $\left(\mathrm{F}_{\mathrm{N} \text { MAX }}\right)$ le taux de photosynthèse mesuré dans les conditions standardisées précisées au $\$ 2$ et dont les variations sont uniquement liées à celles de l'état physiologique des rameaux.

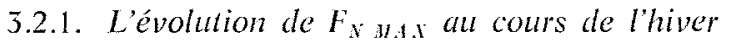

L'évolution de $\mathrm{F}_{\mathrm{N} \text { Mx }}$, en relation avec le régime thermique extérieur, est représentée pour les 3 espèces dans la figure 2 .

Pour l'hiver 1982-1983, qui fut particulièrement doux à Nancy avec une seule période de gel prononcé en février, dans une première approche, l'évolution générale de $F_{X M x}$ ne montre par de différences fondamentales entre les 3 espèces. $F_{N}$ max reste relativement stable en début d'hiver à un niveau avoisinant $7 \mathrm{mg} \mathrm{CO}_{2} . \mathrm{dm}^{-2} \cdot \mathrm{h}^{-1}$ pour les 3 espèces, puis chute de façon importante en relation avec la phase de gel de février, avant de remonter au cours du printemps au niveau des valeurs observées avant février pour le douglas et l'épicéa, mais à un niveau restant nettement en-deçà des valcurs d'avant février pour le sapin. Toujours au cours de cette période printanière on note, après le $1^{\text {er }}$ avril, une diminution nette de la capacité photosynthétique de l'épicéa par rapport à celle du douglas.

L'épicéa a un comportement différent des autres espèces à l'entrée de l'hiver au plan de l'évolution de $\mathrm{F}_{\mathrm{N} M A \mathrm{X}}$ : au cours du mois de novembre et début décembre, la capacité photosynthétique de l'épicéa présente une diminution continue très sensible, d'ailleurs confortée par une valeur de $F_{X \text { max }}$ égale à $9,5 \mathrm{mg} \mathrm{CO}_{2} \cdot \mathrm{dm}^{-2} \cdot \mathrm{h}^{-1}$ obtenue at 5 novembre au cours de mesures préliminaires.

Une telle dépression de $F_{X}$ max, non perceptible pour le douglas, semble atissi exister mais de façon totalement réversible, pour le sapin en décembre et au début de janvier.

\subsubsection{Différences entre espèces}

Faisons remarquer tout d'abord que les différences observées pour les valeurs

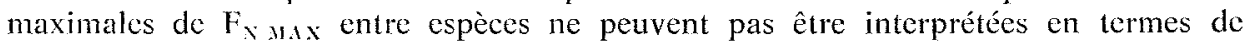
productivité (nécessité pour cela de raisonner à l'échelle du couvert forestier et dans le temps). Ainsi les travaux récents de Gross et Hettesheimer (1983) montrent, pour diverses provenances d'épicéa, une relation négative entre les taux de capacité photosynthétique et les potentialités de production; les différences de production étant alors surtout associées à des différences d'indice foliaire.

Afin de mieux faire ressortir les différences entre espèces dans les variations de $F_{X \ldots \ldots}$ on a représenté (fig. 3) les valeurs de $F_{X \text { m }}$ (exprimées en p. 100 des valeurs maximales observées pour chaque espèce) établies pour le sapin ( $3 \mathrm{~A}$ ) et l'épicéa (3 B) en fonction de celles mesurées pour le douglas et cela pour l'ensemble de la période ćtudiée. La figure $3 \mathrm{~A}$ montre qu'en dehors des points relatifs à la période printanière, il existe une relation nette entre les deux espèces tendant à faire apparaître : 


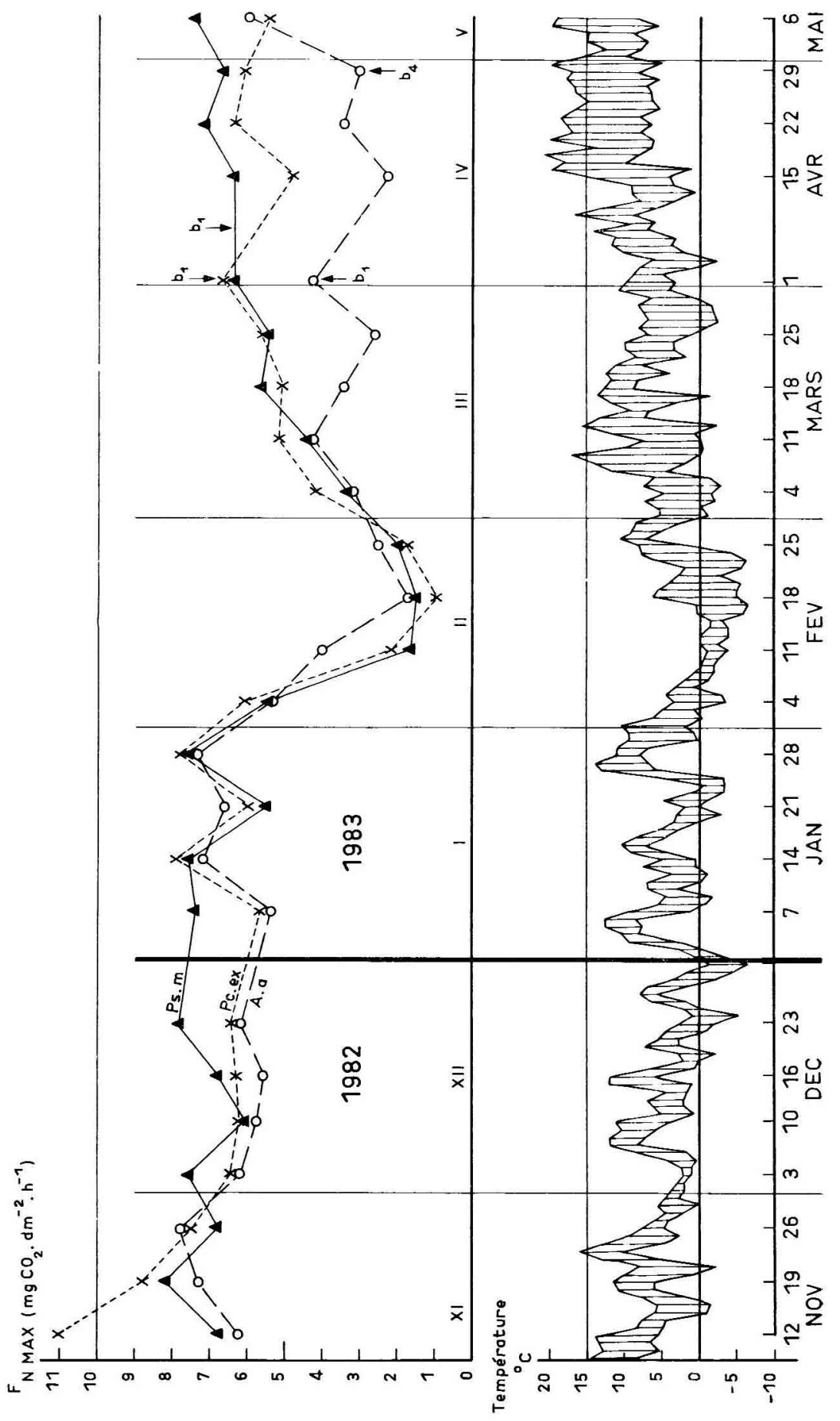


A
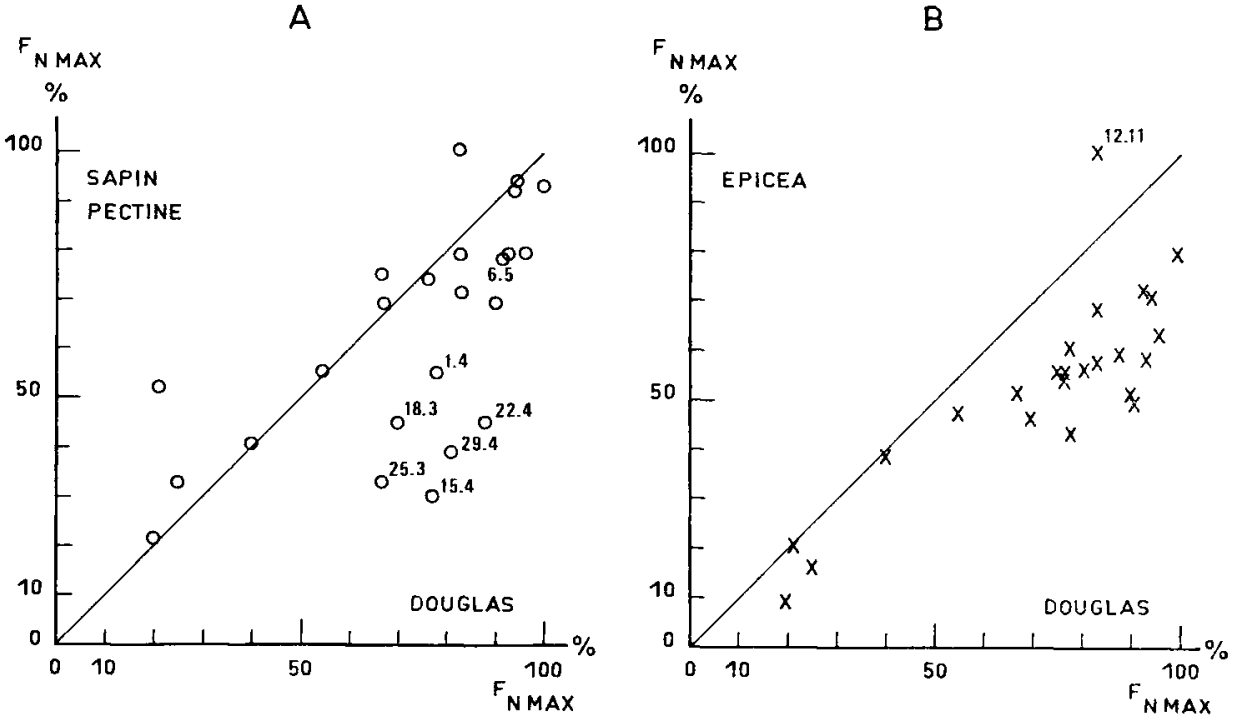

FIG. 3

Relations entre les capacités photosvnthétiques du sapin et du douglas (3 A) et celles de lépicéa et du douglas $(3 B)$.

Les valeurs sont exprimées en p. 100 des valears maximales observées pour chaque espèce.

Comparison of the photosynthesic capacity between silver-fir and douglas-fir (5 A) and between Norway sprace and douglas-fir (3B).

Values are p. 100 of the maximum values for cach species.

- une légère supériorité du douglas sur le sapin pour les $F_{X}$ max élevés (points surtout en dessous de la première bissectrice);

- une supériorité du sapin sur le douglas pour les valeurs relatives de $F_{\mathrm{X} \text { anx }}$ inféricures à 70 p. 100 (points au-dessus de la première bissectrice), c'est-à-dire dans les situations de stress thermiques les plus accusées.

La figure 3 B fait apparaître une supériorité systématique du douglas sur l'épicéa, à relier, bien entendu, à la remarque faite précédemment sur l'évolution de $F_{\mathrm{N} \text { Max }}$ pour l'épicéa à l'entrée dans l'hiver.

FIG. 2

Evolution parallèle au cours de l'hiver 1982-1983, de la capacité photosynthétique $\left(F_{N \text { an }}\right)$ du douglas (Ps.m), du sapin (A.a.) et de l'épicéa (Pc.ex), et du régime thermingue extéricur. Stades da débourrement : $b_{t}=$ début de gonflement des bourgeons. $b_{i}=$ nouvelles aiguilles partiellement libérées.

Changes in the phofosynthetic capacity of douglas-fir (Ps.m), silver-fir (A.a.) and Norway spruce (Pc.ex) during the winter 1982-1983 as related to the outside thermal regime. Bud development states: $b_{1}=$ begin of bad swelling, $b_{i}=$ newly formed needles. 
Au total, les figures $3 \mathrm{~A}$ et $3 \mathrm{~B}$ tendent donc à faire apparaître le classement suivant vis-à-vis du maintien de valeurs élevées de $F_{X \text { m.x }}$ au cours de l'hiver (on fail abstraction pour le sapin des points obtenus durant le printemps) :

$$
\text { Sapin > Douglas > }>\text { Epicéa }
$$

\subsubsection{Analyse des relations entre la capacité photosynthétique et l'histoire thermique des rameanx}

Dans le cadre de travaux antérieurs (GUEHL, 1982) nous avons pu établir la bonne valeur explicative pour $F_{X}$. ax de variables climatiques prenant en considération les températures minimales journalières de quelques journées précédant les mesures de $\mathrm{F}_{\mathrm{N} \text { y.x, }}$, et notamment de :

$$
T_{M H X 1: A}=\frac{1}{6} \underset{i=1}{\stackrel{3}{2}}(4-\mathrm{i}) \mathrm{T}_{\mathrm{MANi}}
$$

où $T_{M x_{j}}$ est la température minimale journalière mesurée sous abri le ième jour avant la mesure. On considère que les journées commencent à 9 heures du matin, heure de prélèvement des rameaux. On a adopté la variable $T_{\text {six }}$..: pour analyser les relations entre $F_{x \text { max }}$ et le régime thermique extérieur (fig. 4).

On examinera dans un premier temps les figures $4 \mathrm{~A}$, B et $\mathrm{C}$ sur lesquelles appa-

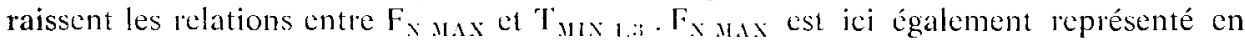
p. 100 de la valeur maximale observée pour chaque espèce. Pour les 3 espéces un groupe de points particuliers (dont les dates correspondantes sont indiquées sur les figures) occupent une position nettement supéricure dans les nuages de points obtenus et créent une dispersion relativement importante pour les valeurs de $F_{X} x_{1} x_{x}$ supérieures à 50 p. 100. Ces points correspondent tous à la période sans gel intense calactérisant l'hiver jusqu'en février, et sont de plus tous situés dans les phases de creux thermiques (cf. fig. 2). Pour ces journées $T_{31 \times 1.3}$ est faible, mais on ne passe pas de façon significative sous le seuil thermique de dépression de $F_{X}$ wax (défini plus clairement ci-dessous

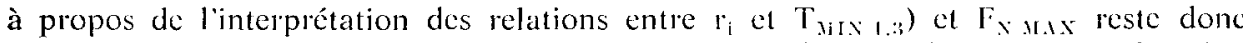
élevé. Cette observation montre les limites de la modélisation de $F_{x} \ldots \ldots x$ en fonction de la seule variable $T_{M N_{1,3}}$ dans le cas d'une structure climatique analogue à celle de la période novembre 1982 - février 1983 où prédominent les températures positives.

En dehors de ces points particuliers et des points obtenus au printemps pour le

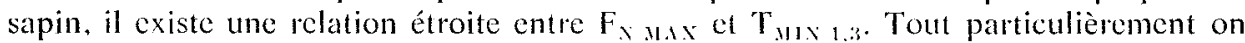

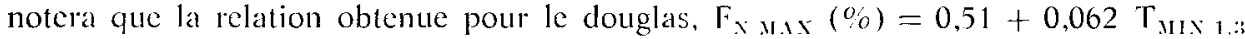
$\left(r^{2}=0,89\right)$, est très voisine de celle déjà trouvée pour la même provenance dans une étude antéticure (Gutull, 1982).

Pour les autres espèces, les équations de régression sont :

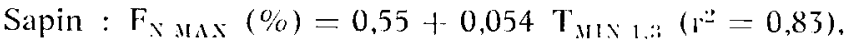

$$
\begin{aligned}
& \text { Epicéa : } F_{x \text { ux }}(\%)=0,37+0,040 T_{11 \ldots 1.3}(1 \%=0,71) \text {. }
\end{aligned}
$$

Ces résultats confortent bien le classement entre espèces proposé à la fin du $\$$ 3.2.2. Les figures $4 \mathrm{~A}^{\prime}$, $\mathrm{B}^{\prime}$ et $\mathrm{C}^{\prime}$ rendent compte des relations existant d'une part entre $\mathbf{r}_{\text {s }}$ et $\mathrm{T}_{\text {MIX 1.3 }}$ et d'autre part entre $r_{i}$ et $T_{M N_{1.3}}$ pour les 3 espèces. Les résistances internes sont pour l'ensemble des mesures nettement supérieures aux résistances stoma- 
$A$
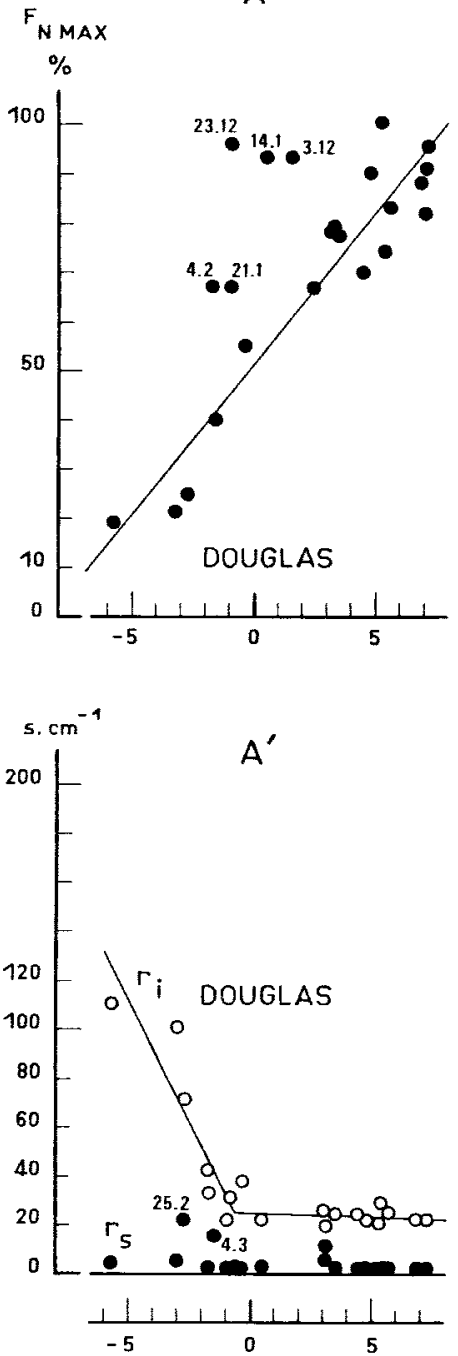

B

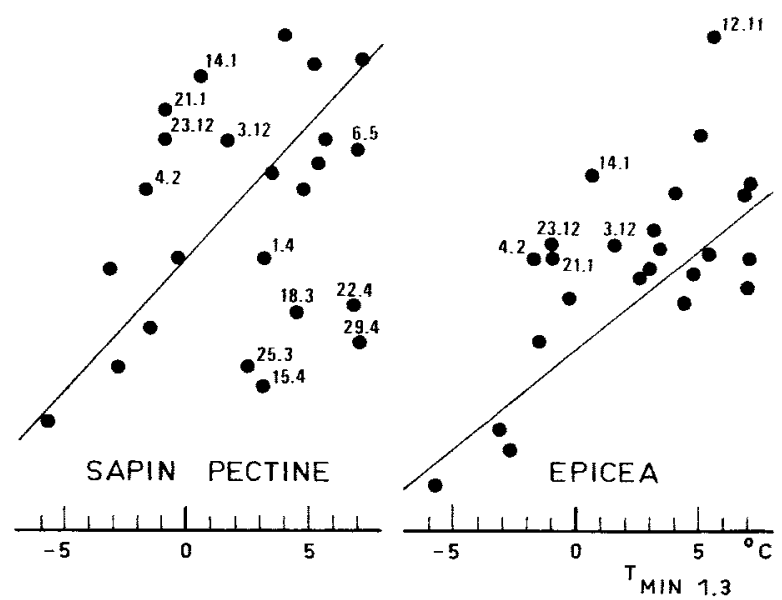

$\mathrm{B}^{\prime}$

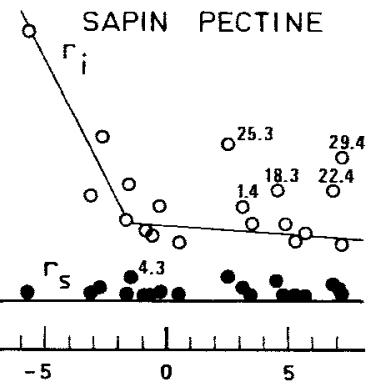

FIG. 4
C

\section{$c^{\prime}$}

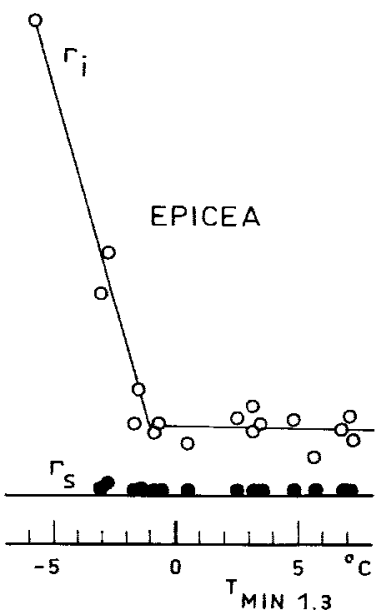

Relations entre la capacité photosynthétique et $T_{M A}$, pour les 3 espèces étudices $(4 A, B, C)$ et relations entre les résistances stomatiques $\left(r_{s}\right)$ et internes $\left(r_{j}\right)$ à la diffusion du $\mathrm{CO}_{3}$

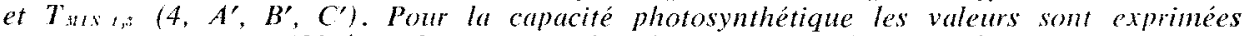
en p. 100 des valeurs maximales observées pour chaque espèce".

Photosynthefic capacity versus $T_{M} t_{r^{3}}$ for the three species $(4 A, B, C)$ and stomatal $\left(r_{s}\right)$ and internal ( $\left.r_{i}\right)$ resistances to the $C O_{2}$ diffusion versus $T_{M} \mathrm{~B}_{1,2}\left(4 A^{\prime}, B^{\prime}, C^{\prime}\right)$.

The photosynthetic capacity values are expressed as p. 100 of the maximum values for each species. 
tiques. Les processus élémentaires de la photosynthèse intégrés dans $r_{i}$ apparaissent donc comme globalement limitants pour la détermination du niveau de $F_{x}{ }_{x \rightarrow x}$ sans qu'il soit possible d'évaluer les rôles limitants respectifs de chacun de ces processus. La limitation en lumière saturante par les processus de diffusion du $\mathrm{CO}_{2}$, (et non par les processus photochimiques), condition nécessaire pour le calcul de $r_{i}(\S 2)$ a été établie par de VITRY (1982) qui a observé le maintien, durant les périodes où $\mathrm{F}_{\mathrm{x} \text { s.x }}$ est affecté par l'effet rémanent des basses températures, d'une relation linéaire croissante entre $F_{X}$ et la concentration en $\mathrm{CO}_{22}$ de l'air entre 0 et $300 \mathrm{vpm}$ pour le douglas et le cèdre.

On note une nette rupture de pente de la relation entre $r_{i}$ et $T_{\text {MIN 1..3 }}$ pour des valeurs de $\mathrm{T}_{M \mathrm{~N}_{1,3}}$ comprises entre -1 et -2 "C pour les 3 espèces. Cette zone thermique singulière peut être interprétée comme le seuil d'endommagement rémanent de la fonction photosynthétique; elle coïncide bien avec la valeur de -1 "C déjà trouvéc pour le douglas (Guent, 1982). Pour l'épicéa la pente de la relation est nettement plus négative sous cette valeur seuil par rapport aux 2 autres espèces et corrélativement la valeur maximale de $r_{i}$ atteinte au 18 févricr $\left(194 \mathrm{~s} . \mathrm{cm}^{-1}\right)$ est nettement la plus importante chez l'épicéa.

Faisons loutefois remarquer que cette forte différence entre espèces pour $r_{\mathrm{i}}$ correspond à des différences de $F_{x}$ max nettement plus faibles (effet amplificateur du passage aux résistances pour les faibles valeurs de $F_{X}$ max).

\section{Discussion}

Parmi les espèces de conifères de grandes différences existent dans les degrés de résistance au gel. Existe-t-il une relation entre cette résistance en termes de survie des organes et la sensibilité au gel de la fonction photosynthétique ? Pour aborder cette question, il est utile de rappeler au préalable quelques résultats trouvés dans la littérature sur la résistance au gel des conifères.

\subsection{Résistance au gel des conifères}

Les conilères des zones tempérée et subarctique de l'Hémisphère Nord peuvent acquérir en hiver des états de résistance importante au gel grâce à des processus d'endurcissement mettant en jeu des modifications graduelles d'ordre physiologique et structural (LARCHER et al., 1973; LARCHER, 1981; LlEVITT, 1980) induites par la diminution de l'héméropériode et des températures à l'entréc de l'hiver (ScHwarz, 1970).

De nombreuses études de résistance au gel menées en conditions de froid artificiel (rcfioidissement lent et progressif) ont permis d'établir le niveau de résistance en termes de survie des différents types de tissus pour un grand nombre d'espèces (voir par exemple DrRrReUdrf, 1978). Les récents travaux de synthèse de LARCHER (1981) et SAKal (1983) proposent une structuration des différences observées à la fois par une typologic des mécanismes d'endommagement et de résistance et par leur interprétation bioclimatique en termes d'adaptation évolutive. 
TABLEAU 1

Niveaux de résistance au froid (températures pour lesquelles on observe un début de nécrose) "t types de résistance des aiguilles de quelques conifères en hiver.

Frost resistance levels (temperatures of first observed needle damages) and resistance types of needles of some conifers during winter.

\begin{tabular}{|c|c|c|c|}
\hline Espèce & Provenance & $\begin{array}{l}\text { Niveau de } \\
\text { résistance au froid }\end{array}$ & $\begin{array}{c}\text { Type } \\
\text { de résistance }\end{array}$ \\
\hline Secunoia sempervirens .... & Am. Nord sous $100 \mathrm{~m}$ & $-10^{\circ} \mathrm{C}$ & $\mathrm{f}$ \\
\hline Pinus pinea ......... & Pourtour méditerranćen & $-10^{\circ} \mathrm{C}$ & 1 \\
\hline Cedrus deodara .. & Himalaya $2500 \mathrm{~m}$ & -15 "C & $\mathrm{t}$ \\
\hline Pinus pinaster ..... & $?$ & $-25^{\prime \prime} \mathrm{C}$ & $t$ \\
\hline Picea sitchensis ....... & Alaska & $-30{ }^{\circ \prime} \mathrm{C}$ & $\mathrm{t}$ \\
\hline Abies nordmanniana .... & Caucase & $-30{ }^{\circ} \mathrm{C}$ & $\mathrm{t}$ \\
\hline Abies alba $\ldots \ldots \ldots \ldots$ & Europe Centrale & $-35{ }^{\circ} \mathrm{C}$ & $\mathrm{t}$ \\
\hline Pinus nigra $\ldots \ldots \ldots \ldots$ & ? & $-40 " \mathrm{C}$ & $\mathrm{t}$ \\
\hline Pseudotsuga menziesii ... & $?$ & -50 "C & $\mathrm{t}$ \\
\hline Picea excelsa ....... & Europe Centrale & $<\ldots 70^{\prime \prime} \mathrm{C}$ & $\mathrm{T}$ \\
\hline Pinus silvestris .... & Europe Centrale & $<-70^{\circ} \mathrm{C}$ & $\mathrm{T}$ \\
\hline Pinus centre. & Europe & $<-70^{\circ} \mathrm{C}$ & $\mathrm{T}$ \\
\hline
\end{tabular}

D'après Larcher (1981) et Sakai (1983) - After Larcher (1981) and SaKal (1983).

$\mathrm{f}$ : espèces sensibles au gel - frost sensitive species.

$\mathrm{t}$ : espèces tolérantes au gel de façon limitée - partially frost tolerant species.

$\mathrm{T}$ : résistance totale au gel - deep frost tolerant species.

On a rassemblé dans le tableau 1 les camactéristiques de résistance indiquées par ces 2 auteurs précités pour un ensemble d'espèces de diverses origines dans la zone tempéréc.

La provenance des espèces n'est malheureusement indiquée que de façon très sommaire et la variabilité intraspécilique, certainement très importante pour les espèces à large distribution (voir par exemple Betlamann \& Schonizacir, 1964 ; Schonbacir \& BEIl LaAn, 1967 pour le Douglas), n’est pas abordéc.

Selon les types de résistances distingués par LARchrR au niveau foliaire, 3 groupes d'espèces apparaissent dans ce tableau concernant uniquement les tissus foliaires.

Lspèces sensibles au gel (f), Pinus pinea. Sequoia sempervirens, la mort des tissus est liée à la formation de glace à l'intérieur des cellules.

Lispèces résistantes au gel de façon limitée (t). La formation de glace à l'intérieur des cellules est évitée (par des états de surfusion, Larciler, 1981). L'endommagement est causé par la déshydratation cellulairc liée à la formation de glace dans les espaces intercellulaires. A lintérieur de ce groupe on note une grande variabilité des niveaux de résistance selon la rigueur hivernale de l'aire d'origine des espèces. Ainsi passe-t-on d'une résistance de $-15^{\circ}$ pour Cedrus deodara à -50 "C pour Pseutdotsuga menziesii 
(il s'agit probablement du douglas bleu plus résistant que la provenance côtière de douglas vert que nous avons étudiée). le sapin pectiné est également à rattacher à ce groupe.

Espèces résistantes au gel de faşon illimilée* (T). Il s'agit d'espèces des genres Pinus, Abies, et Picea (le genre Larix aussi en fait partic pour les parties non foliaires) ayant développé, probablement à partir du miocène tardif (FLorin, 1963 cité par SAKAI, 1983) des espèces dont la résistance au gel permet actuellement la colonisation des zones subarctiques ou de l'étage subalpin où règnent des conditions hivernales extrêmes. La possibilité d'un endurcissement plus important pour ces espèces semble être liée à l'existence d'un rythme endogène de variations saisonnières de la résistance, restant perceptible même en conditions climatiques constantes de phytotron (ScHWarz, 1970). L'épicéa est dans notre étude, représentatif de ce groupe.

\subsection{Effets rémanents des basses températures sur la photosynthèse des conifères}

Les seuils d'endommagement rémanent de la fonction photosynthétique sont bien entendu nettement supérieurs aux valeurs de résistance indiquées ci-dessus, sauf pour les espèces du type $\mathrm{f}$. Pour l'ensemble des espèces étudiées ici, ces seuils sont voisins de $-1,-2{ }^{\circ} \mathrm{C}$. Ces valeurs sont sensiblement supérieures aux températures de formation de glace mises en évidence en laboratoire pour les aiguilles de conifères mêmc en été (-3, - 4 "C ; Larcher, 1973). Larcher (1973) indique pour l'hiver des températures encore nettement plus faibles $\left(-6,-8^{\circ} \mathrm{C}\right)$ pour les mêmes espèces, et Pisek et Kemnitzer (1968) ont pu établir de façon très claire par application de froids artificiels à des rameaux coupés prélevés à l'extérieur (satin pectiné, provenance continentale de basse montagne en Autriche) que les seuils de dépression de la capacité photosynthétique peuvent être nettement inférieurs à ces valeurs (tolérance au gel). Mais sans doute s'agit-il là de valeurs extrêmes traduisant des conditions d'endurcissement profond, réalisées dans un contexte climatique où les possibilités de photosynthèse hivernale sont très faibles, et probablement non atteintes en situation de plaine en France.

Dans une situation climatique plus proche de celle décrite dans la figure 2 , Neılson et al. (1972) ont trouvé pour l'épicéa de Sitka, à l'extérieur, une diminution de l'activité photosynthétique dès $-1^{\circ} \mathrm{C}$ alors qu'en chambre climatisée les mêmes plants n'étaient pas affectés à $-5^{\circ} \mathrm{C}$ (les plants étaient exposés au froid durant une nuit). Nous avons déjà évoqué (GueHL, 1982), les écarts nocturnes négatifs entre les températures de feuille et la température de l'air, écarts liés au rayonnement de grande longueur d'onde, pour expliquer de telles différences suivant les conditions méthodologiques. Une telle explication pourrait aussi s'appliquer à l'écart entre les seuils thermiques de dépression de la photosynthèse et les températures (établies en laboratoire) de formation de glace dans les espaces intercellulaires. Mais des écarts de 4 , voire 5 ou $6^{\circ} \mathrm{C}$ peuvent-ils être expliqués ainsi ?

Pharis et al. (1970) ont mis en évidence une dépression de la capacité photosynthétique de plants de douglas endurcis au froid placés dans une chambre climatisée à $-2{ }^{\circ} \mathrm{C}$ durant la nuit au cours d'une expérience s'étendant sur plusieurs jours, et dans des conditions où l'effet thermique décrit ci-dessus ne peut être évoqué.

* Du moins pour les températures pouvant exister à la surface du globe. 
Les phénomènes de photo-inhibition (action conjointe d'éclairements forts et de températures basses mais non forcément négatives) mis en avant par Oouist (1983) pour expliquer les fortes dépressions hivernales de capacité photosynthétique, et qui pourraient se produire à des températures supérieures à celles entraînant un gel intracellulaire, ne nous paraissent pas pouvoir être incriminés ici ; en effet :

- les variations de $F_{X}$ max apparaissent comme régies par les processus de diffusion du $\mathrm{CO}_{2}$ ( $\mathrm{cl}$. \$ 3.2 .3 .) ;

- des dépressions importantes de $F_{\text {Max }}$ peuvent être notées en cours de périodes où les températures diurnes restent largement positives (Guent, 1982).

On peut alors se demander si les abaissements cryoscopiques importants (pouvant également englober un terme de surfusion non stable?) constatés en laboratoire pour des expérimentations de courte durée sont transposables à l'extérieur ou à des darées d'expérimentation plus longues.

La dépression de la capacité photosynthétique liée aux températures inféri: zures aux seuils thermiques mis en évidence au $\$ 3.2 .3$. semble la plus importante pour l'épicéa, malgré sa plus grande capacité de résistance (survic) à des températures nettement plus basses.

Mais la différence la plus nette entre les espèces étudiées ici est constituée par la chute de $F_{x}$ max à l'entrée de l'hiver, apparaissant de façon importante chez l'épicéa. Cette chute est-elle liée à l'endurcissement aux basses températures, et est-elle spécifique des espèces de type $T$ ? Les connaissances restent trop partielles à l'heure actuelle pour confirmer une telle opposition systématique, mais certains résultats méritent d'être cités.

Ainsi FRY \& PHILLIPS (1977) n'ont pu mettre en évidence aucune dépression de $F_{x, \ldots x}$ en relation avec les facteurs climatiques dans les conditions hivernales clémentes des lles Britanniques pour Picea sitchensis. Tsuga heterophylla et Abies grandis (toutes cspéces de type 1). OOUIST \& MARTiN (1980) ont noté pour le pin sylvestre une nette dépression de $\mathrm{F}_{\mathrm{X} \text { ux }}$, non expliquée par les processus photochimiques, sous l'action de basses températures non négatives simulant l'entrée dans l'hiver. Schwarz (1971) a pu élablir que dans les conditions rigoureuses d'altilude dans les montagnes rocheuses, la chute de photosynthèse à l'entrée de l'hiver, le rétablissement au cours du printemps, et les potentialités de réactivation par des températures positives en laboratoire au cours de l'hiver sont nettement plus favorables pour le douglas (espèce t) que pour Picea glataca et Pinus contorta (espèces T).

Dans les conditions climatiques décrites par la figure 2, le rélablissement de $F_{\mathrm{N}}$ ax après le coup de froid de février est dans un premicr temps identique pour les trois espèces. Pour le douglas, on enregistre des valeurs équivalentes au niveau d'avant l'hiver dès le mois d'avril. Pour le sapin d'abord (à partir du 11 mars) puis pour

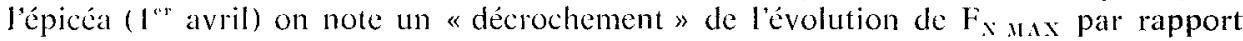
au douglas. Ces comportements sont à notre avis à relier à l'activité respiratoire croissante des bourgeons au cours des périodes de pré-débourrement provoquant une diminution de loptimum thermique des échanges globaux de CO.2 du rameau (GueHL, 1982), on notera d'ailleurs que l'ordre de succession des décrochements de $F_{N}$ max respecte celui des débourrements (fig. 2). Les mesures que nous avons effectuées à 20 "C ont donc été faites dans des conditions supra-optimales qui d'ailleurs ne sont approchées à l'extérieur qu'à la sortie du mois d'avril. L'expression de la dépression de la photosynthèse pour le sapin est donc certainement nettement inférieure en conditions naturelles à ce qu'il apparaît sur la figure 2 . 


\subsection{Photosynthèse et température actuelle}

Un second type de relation entre la photosynthèse et le régime thermique est constitué par les relations $F_{X}(1)$. Au cours de l'hiver on ne note pas de différences très significatives entre les espèces. La photosynthèse est affecté de façon importante par les températures inférieures à 5 "C. Lc point de compensation inférieur, égal à $-3^{\circ} \mathrm{C}$ pour le douglas (fermeture stomatique?), scmble inférieur $\left(-5^{\circ} \mathrm{C}\right.$ ) pour les autres espèces. Pour ces dernières espèces ce point singulier pourrait correspondre au point de congélation dans les espaces intercellulaires (LARCHER, 1973). La différence entre espèces serait alors relativisée par les réserves faites au $\$ 4.2$. sur la transposition des températures de congélation établies en laboratoire aux conditions in situ.

La courbe obtenue en été pour le sapin présente un décalage avec la courbe hivernale montrant un phénomène d'ajustement saisonnier déjà mis en évidence par Neilson et al. (1972) pour l'épicéa de Sitka. Par contre, pour l'ensemble des conifères que nous avons étudiés, il ne semble pas exister un mécanisme d'ajustement chronologique à court terme aux fluctuations thermiques extérieures tel que cela a pu être mis en évidence pour Eucalyptus pauciflora (Slatyer \& Morrow, 1977). II faut remarquer qu'en termes d'adaptation au régime thermique une courbe hivernale à large domaine optimal s'étendant vers les basses températures constitue une stratégie d'acclimatation au moins aussi performante que l'existence d'un ajustement rapide aux fluctuations thermiques s'effectuant avec un déphasage de plusieurs jours et dont l'efficacité est limitée vers les basses températures.

\section{Conclusions}

Nous avons pu mettre en évidence pour les espèces étudiées l'existence de potentialités importantes de photosynthèse hivernale dans une situation favorable d'hiver doux. Des différences sont toutefois apparues entre espèces tendant à dégager deux grands types d'évolution saisonnière de la photosynthèse.

Les espèces ou provenances originaires des zones tempérées à l'hiver relativement doux (douglas, sapin) présentent une photosynthèse hivernale uniquement affectée par les variations "accidentelles» liées aux stress thermiques. Les caractéristiques de résistance à ces stress (tolérance et surtout rétablissement après les stress) apparaissent comme favorables à l'existence d'une photosynthèse hivernale importante dans des conditions climatiques proches de celles de leur aire d'origine.

Les espèces ou provenances originaires des zones tempérées à hiver relativement nentales ou montagnardes à hivers rudes (épicéa) ne se caractérisent pas par une plus grande résistance de la photosynthèse aux basses températures, en dépit de leur possibilité de survic en conditions de stress extrêmes. De plus, aux variations accidentelles lićes aux stress semble se superposer une tendance saisonnière de dépression de la photosynthèse en hiver à relier au rythme interne de variations saisonnières de l'endurcissement au froid déjà connu pour ses espèces. Une telle rythmicité saisonnière affecte-t-elle aussi d'autres activités physiologiques telles que la croissance et l'activité racinaire, les relations hydriques? Elle pourrait alors contribuer à expliquer, dans les zones à hivers doux, des différences de production entre espèces ou provenances de zones à hivers froids et celles originaires de zones à hivers plus cléments. 
Un 3" groupe d'espèces pour lequel il serait intéressant de connaître les caractéristiques d'adaptation aux basses températures de la photosynthèse est celui des espèces du groupe t moins résistantes au gel que le douglas et le sapin (par exemple en France, le pin maritime ou les cèdres).

Ces espèces occupent en France des zones où le climat hivernal, lumineux notamment, est plus favorable que dans les zones occupées par le douglas ou le sapin. Une moindre résistance au gel reste-t-elle pour ces espèces compatible avec un bon comportement de la photosynthèse aux basses températures? Les premiers éléments de réponse à cette question réunis pour les cèdres (Ducrty, 1981 b ; de VITRY, 1982) restent encore trop partiels pour qu'un schéma général puisse être proposé.

\section{Summary}

Comparative study of the winter photosynthetic potential of three evergreen conifers of the temperate zone (Pseudotsuga menziesii Mirb., Abies alba Mill. and Picea excelsa Link.)

The winter-course of photosynthesis was studied on shoots of douglas fir, silver fir and Norway spruce cut from outside stands.

The pattern of photosynthesic response to temperature was similar for the 3 species with a wide optimal range from 5 to $25^{\circ} \mathrm{C}$. Low temperature limits were however -3 " $\mathrm{C}$ for douglas and -5 " $\mathrm{C}$ for fir and spruce.

Threshold values for lasting depressive effects of low temperatures were of about -1 , $-2{ }^{\circ} \mathrm{C}$, irrespective of species.

Douglas fir and silver fir, partially frost-resistant species and originating from mild winter climatic areas, exhibited a winter-course of photosynthesis affected only by changes in the thermal history of the shoots. In contrast, for the Norway sprace, for which a continental deep frost-resistant provenance was used, a more complex evolution pattern appeared, superposing a seasonal trend (winter depression) on the short term variations due to the thermal history.

Discussions on the relevance of an extrapolation of the above-mentionned patterns with respect to the frost resistance characteristies are made.

\section{Références bibliographiques}

Bellmann E., Schonbaru H., 1974. Erfolgsaussichten der Ausleseziichtung auf Frostresistenz bei der grünen Douglasic (Pseudotsuga menziesii (Mirb.), Franco.). Arch. f. Forstw., 13, 307-331.

Chartier P., Bethlinod O., 1977. La productivité à l'échelle de la feuille. In : «Les processus de la production végétale primaire », MoYse A., Gauthier-Villars, 77-112.

Derreudre J., 1978. Effets de divers types de refroidissements sur la teneur en eau et sur la résistance au gel des bourgeons de rameaux d'épicéa en vie ralentie. Physiol. Vég., 16 (3), 469-489.

DE Vitry C.. 1982. Photosynthese hivernale du Cèdre (Cedrus libani I oud. et Cedrus atlantica Maneti) et du Douglas (Pseudotsuga menziesii Mirb.). D.E.A. de Physiologic végétale, Université Nancy I. Document Station Sylviculture et Production. C.N.R.F. Nancy. 47 pp. + Annexes. 
Ducriy M., 1981 a. Etude bioclimatique d’une futaic feuillue (Fagus silvatica L. et Quercus sessiliflora Salisb.) de l'Est de la France. III - Potentialités photosynthétiques des feuilles à différentes hauteurs dans le peuplement. Ann. Sci. For., 1981, 38 (1), $71-87$.

Ducrey M., 1981 b. Action des basses températures hivernales sur la photosynthèse du cèdre et du douglas. Ann. Sci. For., 38 (3), 317-329.

Florin R., 1963. The distribution of Conifer and Taxad genera in time and space. Acta Horti. Bergiani, 20, 121-312.

Fry D.J., PHillips I.D.J., 1977. Photosynthesis of conifers in relation to annual growth cycles and dry matter production. II. Scasonal photosynthetic capacity, and mesophyll ultrastructure in Abies grandis, Picea sitchensis, Tsuga heterophylla and Larix leptolepis growing in S.W. England. Plysiol. Plant, 40, 300-306.

GRoss K., Hetreshlimer W., 1983. Vergleichende Gaswechselmessungen an schncll und langsam wachsenden 18-jährigen Fichten unterschiedlicher geographischer Provenienz. Allg. Forst.-It. J.-Ztg, 147, 169-177.

Guehl J.M., 1982. Potenticl de photosynthèse hivernale du douglas (Pseudotsuga menziesii Mirb.) en relation avec le régime thermique. Aln. Sci. For., 39 (3), 239-258.

I.ANidsB1:RGi J J., Ludrow M.M., 1970. A technique for detcrmining resistance to mass transfer through the boundary layers of plants with complex structure. J. Appl. Ecol., 7 (1), 76-86.

LArcher W., 1981. Resistenzphysiologische Giundlagen der evolutiven Käleatkilimatisation von Sprossflanzen. Pl. Syst. Evol., 137, 145-180.

Larchler W., Herbir V., Santarius K.A., 1973. Limiting temperatures for life functions. In : T'mperature and life, PRichlT H. et al., Springer Verlag, 195-263.

1.L.VITT J., 1980. Responses of plants to environmental stresses. Vol. 1 : Chilling, freezing, and high temperature stresses. Academic Press, $470 \mathrm{p}$.

Ni:Itson R.E., Ludlow M.M., Jarvis P.G., 1972. Photosynthesis in Sitka Spruce (Picét silchensis (Bong.) CARr.). I1. Response to temperature. J. Appl. Ecol., 9, 72l-745.

Oquist G., 1983. Effects of low temperature on photosynthesis. Plant Cell. Envir., 6, 281-300.

Oquist G., MarTin B., 1980. Inhibition of photosynthetic electron transport and formation of inactive chlorophyll on winter stressed Pinus silvestris. Physiol. Plant., 48, 33-38.

Pitaris R.P., Hellmers H., Schuurmans F., 1970. Effects ol subfreezing temperatures on photosynthesis of evergreen conifers under controlled environment conditions. Photosyuthetica, 4 (4), 273-279.

Pisek A., Kranniziz R., 1968. Der Einfluss von Frost auf die Photosynthese der Weisstanne (Abies alba Mill.). Flora, Abt. B., 157, 314-326.

Pistik A., Larcher W., Vligis A., Napr-Sinn K., 1973. The normal temperature range. In : Temperature and life, Precit H. et al., Springer Verlag, 102-195.

SAKA A., 1983. Comparative study on reezing resistance of conifers with special reference to cold adaptation and its evolutive aspects. Can. J. For., 61 (9), 2323-23.32.

Schonbacil H., Beilman E., 1967. Frostresistenz der Nachkommenschaften von Kreuzungen "griiner» und «blauer» Formen der Douglasie (Pseudotsuga menziesii (Mirb.) Franco). Arch. Forstives. 16, 707-711.

Sc HWakz W., 1970. Der Einfluss der Photoperiode auf das Austreiben, die Frosthärte und die Hitzeresistenz von Zirben und Alpenrosen. Flora, 159, 258-285.

Schwarz. W., 1971. Das Photosynthesevermögen einiger Immergrüner während des Winters und seine Reahtivicrungsgeschwindigkeit nach scharfen Frösten. Ber. Dtsch. Bot. Ges., 84, H. 10, $585-594$.

Si.ATyir R.O., Morrow P.A., 1977. Altitudinal vartations in the photosynthetic characteristics of snow gum, litcalyptes pateiflora Sieb. ex Spreng. I. Seasonal changes under field conditions in the snowy mountains area of south-eastern Australiat. Aust. J. Bot.. $25,1-20$.

Trangullini W., 1979. Dry matter production of trees at Timberline. In : Physiological ecology of the alpine Timberline. Springer Verlag, 39-80.

ZIilaWSKI W., Göral. I., 1966. Seasonal changes in the photosynthesis rate of Scots pine (Pinus silvestris L.) seedlings grown from seed of various provenances. Acta Societ. Bot. Pol., 35 (4), 587-598. 